\title{
Association between eating behavior, anthropometric and biochemical measurements, and pyy hormone levels in obese adolescents in outpatient care
}

\author{
Simone P. Fernandes ( $\nabla$ simone.p.fernandes@gmail.com ) \\ Janine Alessi \\ Universidade Federal do Rio Grande do Sul Instituto de Ciencias Basicas da Saude \\ https://orcid.org/0000-0003-4311-3307

\section{Zilda de Albuquerque Santos} \\ Universidade Federal do Rio Grande do Sul Instituto de Ciencias Basicas da Saude \\ Elza Daniel de Mello \\ Universidade Federal do Rio Grande do Sul Instituto de Ciencias Basicas da Saude
}

\section{Research}

Keywords: Feeding Behavior, Weight loss, Peptide YY, Pediatric obesity, adolescente,Comprehensive Health Care

Posted Date: December 12th, 2019

DOI: https://doi.org/10.21203/rs.2.18644/v1

License: (c) (i) This work is licensed under a Creative Commons Attribution 4.0 International License. Read Full License 


\section{Abstract}

Background: Food intake and energy expenditure, as well as short-term satiety signals, can be regulated by the peptide tyrosine tyrosine (PYY) secreted by the gastrointestinal tract. It is also unknown if changes in PYY hormone levels lead to changes in feeding behavior or in body composition. More comprehensive investigation of serum regulation of gut-derived hormone signals, with behavioral and clinical outcomes, are needed to understand the consequences of deregulating the appetite of obese adolescents. Objective: To evaluate the eating behavior of obese adolescents and its association with biochemical, anthropometric and PYY measures.

Methods: 51 obese adolescents received counseling for weight management at 12 monthly appointments. Fasting serum PYY levels, total cholesterol (TC), high density lipoprotein cholesterol (HDLC), triglycerides (TG), low density lipoprotein cholesterol (LDL-C), insulin and glucose levels, waist circumference (WC) and results from the Three-Factor Eating Questionnaire (TFEQ-21) were assessed.

Results: Over one year there was a significant increase in PYY levels $(p=0.026)$; reduction in TC $(p=$ $0.003), T G(p=0.022), B M I(p=0.002), B M I z$-score $(p<0.001)$ and WC $(p=0.003)$. During this period there was a decrease in the Uncontrolled Eating score (UE), illustrating that adolescents displayed more self-control $(p=0.008)$ at the end of the study; however, this result was independent of BMI and BMI zscore $(p=0.407)$. The reduction in UE was associated with a significant improvement in insulin levels ( $r s$ $=0.326 ; p=0.020)$. The reduction in UE was also associated with lower levels of blood glucose $(r=0.332$; $p=0.017)$, and the increase of cognitive restriction, with the reduction of insulin insulin ( $r s=-0.294 p=$ $0.036)$ and TG $(r=-0.368 ; p=0.008)$ and an increase in Cognitive Restraint.

Conclusions: Our results show that after a year of monitoring weight loss, adolescents had more controlled eating behaviors, increased PYY levels, and reduced weights.

\section{Background And Relevance Of The Study}

Food intake and energy expenditure, as well as short-term satiety signals, can be regulated by the peptide tyrosine tyrosine (PYY) secreted by the gastrointestinal tract. It is also unknown if changes in PYY hormone levels lead to changes in feeding behavior or in body composition. More comprehensive investigation of serum regulation of gut-derived hormone signals, with behavioral and clinical outcomes, are needed to understand the consequences of deregulating the appetite of obese adolescents.

The clinical intervention with nutritional and behavioral guidelines proposed in this study showed that adolescents with obesity, who change their sedentary behavior and adhere to a balanced diet, present weight reduction, increased PYY levels and a more controlled eating attitude. Over the course of a year, was also a reduction in the score of uncontrolled eating behavior, body mass index, body mass and abdominal circumference.

\section{Introduction}


Obesity is a global concern and many strategies and solutions have been sought to prevent and treat it in the long term. Worldwide, the prevalence of overweight and obesity combined increased to $47.1 \%$ among children between 1980 and 2013 (1). In 2013, about 23.8\% and 22.6\% of boys and girls, respectively, were overweight or obese in developed countries, while 12.9 and $13.4 \%$ of boys and girls were overweight or obese in developing countries, respectively (1). About $14.1 \%$ of children and adolescents in Brazil are obese (2).

Eating behavior (EB) contributes to body weight regulation and is influenced by several factors (biological, economic, social and psychological). According to Stunkard and Messick (1985) EB can be evaluated in different dimensions, including Cognitive Restraint (CR), Uncontrolled Eating (UE) and Emotional Eating (EE), all which involve organic sensations of hunger and satiety, motivational states, and a need for energy. The latter is a metabolic and physiological process, coordinated by the central and peripheral nervous system (3).

$\mathrm{CR}$ is a mental condition in which the individual voluntarily avoids fattening foods, reduces portion sizes, denies hunger signals, stops eating or skips meals while still hungry, in order to lose weight or change body shape. ED is characterized by a loss of control over food where a person is unable to control what or how much is being consumed. EE is characterized by binge eating in response to negative emotional states such as loneliness, anxiety and depression (4).

The complexity of eating behavior has driven the search to identify mechanisms that influence eating habits. Studying the different dimensions of EB during lifestyle interventions can serve as a tool to identify causes of obesity and track treatment strategies for obese people, especially in the severely obese (5).

Given the lack of research on EB, the objectives of our study were to evaluate the EB dimensions of Brazilian obese adolescents and determine its association with gender, biochemical parameters, anthropometric data, and PYY intestinal hormone levels. The hypothesis of this study is that individuals with increased PYY levels due to participation in a lifestyle intervention, change eating behavior over time.

\section{Materials And Methods}

A prospective cohort study was conducted over one year with obese adolescents receiving outpatient treatment at a public hospital in southern Brazil. We included adolescents who were at least 10 years old and had a pubertal stage greater than I (6). Obesity was defined as a body mass index (BMI) equal or greater than the 97th percentile, and severe obesity was defined as a BMI greater than the 99.9th percentile, for gender and age (7).

All participants attended monthly appointments, where anthropometric measurements (weight, height, waist circumference (WC)) and a food history were completed. This information was discussed by an interdisciplinary team and a plan of action was defined with nutritional recommendations and 
encouragements to reduce sedentary behavior, as suggested by Mello et al. (2004), Barlow (2007) and Fernandes et al. (2012) (8-10).

Among the nutritional recommendations proposed were: reduce and / or remove sugary drinks (artificial juices and soft drinks) and unhealthy foods (sandwich cookies, snacks, pastries,chocolates, etc) from the diet, eat 3 units of fruit / day, eat dairy foods (milk, yogurt, or two slices of cheese) at least $1 x$ / day, reduce the portion size of foods commonly consumed, eat breakfast, have meals at the table, chew food more thoroughly, and set a time to eat the main meals. Stimuli to reduce sedentary behavior were suggested: limit screen time (TV, tablet, phone, computer); engage in more physical activity (volleyball, soccer, handball, dancing, walking, playing with friends).

At T0 (right before the first appointment), T1 (24 weeks) and T2 (48 weeks) fasting blood samples were collected to quantitate: total cholesterol (TC), high density lipoprotein cholesterol (HDL-C), triglycerides (TG), blood glucose, insulin and the hormone $\mathrm{PYY}_{3-36}$. An electrical impedance test (BioDynamics450) assessed percent body fat (\% BF) and basal metabolic rate (BMR). Insulin and glucose data were used to calculate homeostasis model assessment (HOMA-IR), and TC and TG were used to calculated lowdensity cholesterol (LDL-C). Then, in a private room, study participants answered a 21-item questionnaire called the Three-Factor Eating Questionnaire (TFEQ21). PYY -36 was determined by ELISA (EnzymeLinked Immunosorbent Assay) using Phoenix commercial kits.

\section{DATA ANALYSIS}

In order to compare T0, T1 and T2, analysis of variance (ANOVA) tests were applied for repeated measures, complemented by Bonferroni or Friedman test in conjunction with the Wilcoxon test. Associations between variables were evaluated by Pearson or Spearman correlation coefficients. The TFEQ21 behavior scales were measured ordinally, and we used Spearman correlations for descriptive purposes. Descriptive statistics of patient characteristics were tabulated by group and included the mean and standard deviation for continuous variables and frequency for categorical variables.

Statistical analyses were performed using SPSS, V21.0 and significance was set at $p<0.05$. All study procedures were reviewed and approved by the ethics committee of the Hospital de Clínicas de Porto Alegre, under protocol 130190.

\section{Results}

The mean age of the 51 adolescents was $12.02 \pm 0.9$ years. Twenty-nine (56.9\%) were female and more than half $(58.82 \%)$ were severely obese. When we compared the degree of obesity with EB scores and metabolic values at baseline (T0), there was no significant difference between obesity groups. However, the severely obese participants had larger WC $(p=0.035)$.

Over one year there was a significant increase in levels of the gut hormone PYY $(p=0.026)$; and a decrease in TC $(p=0.003), T G(p=0.022), B M I(p=0.002)$, BMI z-score $(p<0.001)$ and WC $(p=0.003)$. 
There was also a decrease in the UE behavior score $(p=0.008)($ Table 1$)$.

Table 1

Comparison between study variables at $\mathrm{T} 0, \mathrm{~T} 1$ and $\mathrm{T} 2$.

\begin{tabular}{|c|c|c|c|c|}
\hline Variables & TO & $\mathrm{T} 1$ & T2 & $\mathrm{p}$ \\
\hline PYY pg/mL* & $55.9 \pm 17.4^{\mathrm{a}}$ & $58.2 \pm 16.6^{a b}$ & $61.2 \pm 17.7^{b}$ & 0.026 \\
\hline $\mathrm{TC} \mathrm{mg} / \mathrm{dL}^{*}$ & $163.6 \pm 29.4^{b}$ & $156.9 \pm 24.4^{\mathrm{ab}}$ & $151.0 \pm 22.5^{a}$ & 0.003 \\
\hline $\mathrm{TG} \mathrm{mg} / \mathrm{dL}^{*}$ & $99.7 \pm 46.1^{a b}$ & $101.9 \pm 43.5^{b}$ & $88.6 \pm 32.8^{\mathrm{a}}$ & 0.022 \\
\hline LDL-c mg/dL* & $100.0 \pm 22.0$ & $96.3 \pm 20.0$ & $93.3 \pm 18.3$ & 0.058 \\
\hline Insulin ** & $19.3(15.2-27.9)$ & $18.7(14.1-26.3)$ & $17.3(12.9-24.3)$ & 0.052 \\
\hline $\mathrm{BMI} \mathrm{Kg} / \mathrm{m}^{2^{\star}}$ & $29.6 \pm 4.4^{b}$ & $28.8 \pm 4.5^{\mathrm{ab}}$ & $28.6 \pm 5.3^{a}$ & 0.002 \\
\hline BMI z-score* & $3.35 \pm 0.88^{c}$ & $3.03 \pm 0.80^{b}$ & $2.80 \pm 0.89^{a}$ & $<0.001$ \\
\hline $\mathrm{WC} \mathrm{cm*}$ & $93.0 \pm 11.3^{b}$ & $90.8 \pm 10.4^{\mathrm{a}}$ & $90.2 \pm 12.1^{a}$ & 0.003 \\
\hline$\% \mathrm{FM}$ * & $32.9 \pm 6.0$ & $31.9 \pm 5.9$ & $32.3 \pm 6.2$ & 0.340 \\
\hline UE score* & $61.9 \pm 12.5^{b}$ & $60.7 \pm 11.2^{b}$ & $55.8 \pm 15.5^{\mathrm{a}}$ & 0.008 \\
\hline CR score* & $42.8 \pm 18.5$ & $46.8 \pm 16.3$ & $45.7 \pm 14.3$ & 0.104 \\
\hline EE score ${ }^{\star \star}$ & $33.3(16.7-50)$ & $27.8(16.7-55.6)$ & $27.8(16.7-44.4)$ & 0.085 \\
\hline \multicolumn{5}{|c|}{ * described as mean $\pm S D * \star$ median (percentiles $25-75$ ) } \\
\hline \multicolumn{5}{|c|}{$a, b$ Same letters indicate no difference by Bonferroni test at $5 \%$ significance } \\
\hline \multicolumn{5}{|c|}{$\begin{array}{l}\mathrm{BMI}=\text { body mass index; } \mathrm{UE}=\text { uncontrolled eating; } \mathrm{CR}=\text { cognitive restraint; } \mathrm{EE}=\text { emotional eating; } \mathrm{PYY} \\
=\text { = peptide } \mathrm{YY} ; \mathrm{TC}=\text { total cholesterol; } \mathrm{TG}=\text { triglyceride; } \mathrm{LDL}-\mathrm{C}=\text { low density lipoprotein cholesterol; } \mathrm{BMR} \\
=\text { basal metabolic rate; } \mathrm{FM} \%=\text { fat mass percentage; } \mathrm{WC}=\text { waist circumference. }\end{array}$} \\
\hline
\end{tabular}

Over a year, 21 patients (41.2\%) reduced their excess weight by more than $0.5 \mathrm{BMI} z$-score. After one year of intervention, the decrease in UE was associated with a significant decrease in insulin levels $\left(r_{s}=0,326\right.$; $p=0,020)$; the decrease in EE was associated with a decrease in glycaemia $(r=0,332 ; p=0,017)$ and the increase in CR was associated with a reduction in insulin $\left(r_{s}=-0,294 ; p=0,036\right)$ and TG $(r=-0,368 ; p=$ 0,008) (Table 2). 
Table 2

Association between eating behaviors and biochemical, anthropometric parameters.

\begin{tabular}{|c|c|c|c|c|}
\hline Associations & TO & $\mathrm{T} 1$ & $\mathrm{~T} 2$ & T0-T2 $(\Delta)$ \\
\hline UE vs HDL-c & -0.064 & -0.134 & $-0.304^{*}$ & -0.157 \\
\hline UE vs TG & $0.287^{*}$ & 0.273 & 0.125 & 0.047 \\
\hline UE vs TC & 0.168 & 0.033 & -0.045 & 0.005 \\
\hline UE vs LDL-c & 0.146 & -0.041 & -0.014 & -0.128 \\
\hline UE vs Glucose & 0.024 & -0.093 & -0.077 & 0.210 \\
\hline UE vs Insulin & $0.285^{\star}$ & 0.214 & 0.199 & $0.326^{*}$ \\
\hline UE vs WC & 0.244 & 0.159 & -0.013 & -0.122 \\
\hline EE vs HDL-c & 0.250 & -0.096 & 0.034 & 0.064 \\
\hline EE vs TG & 0.149 & 0.133 & 0.199 & 0.063 \\
\hline EE vs TC & $0.319 *$ & 0.165 & 0.138 & 0.012 \\
\hline EE vs LDL-c & 0.235 & 0.101 & 0.197 & -0.012 \\
\hline EE vs Glucose & 0.155 & 0.207 & 0.033 & $0.332^{*}$ \\
\hline EE vs Insulin & -0.098 & 0.017 & 0.037 & 0.145 \\
\hline EE vs WC & 0.266 & $0.300 *$ & 0.160 & -0.073 \\
\hline CR vs HDL-C & -0.016 & 0.039 & -0.011 & -0.082 \\
\hline CR vs TG & $-0.405^{\star \star}$ & -0.184 & -0.112 & $-0.368^{\star *}$ \\
\hline CR vs TC & $-0.312^{\star}$ & -0.156 & -0.125 & -0.063 \\
\hline CR vs LDL-c & -0.205 & -0.034 & -0.237 & -0.076 \\
\hline CR vs Glucose & -0.098 & -0.104 & 0.127 & -0.011 \\
\hline CR vs Insulin & $-0.346^{*}$ & -0.139 & -0.083 & $-0.294^{\star}$ \\
\hline CR vs WC & -0.031 & -0.015 & -0.014 & -0.032 \\
\hline \multicolumn{5}{|c|}{$\begin{array}{l}\left.* \mathrm{p}<0.05^{* *} \mathrm{p}<0.01-\text { Significant association between eating behavior (ED, EE and } C R\right) \text { with } \\
\text { biochemical parameter by Pearson }(r) \text { or Spearman }\left(r_{s}\right) \text { correlation coefficients. }\end{array}$} \\
\hline
\end{tabular}




\begin{tabular}{|c|c|c|c|c|}
\hline Associations & TO & $\mathrm{T} 1$ & T2 & T0-T2 $(\Delta)$ \\
\hline \multicolumn{5}{|c|}{$\begin{array}{l}* p<0.05 * * p<0.01-\text { Significant association between eating behavior (ED, EE and CR) with } \\
\text { biochemical parameter by Pearson }(r) \text { or Spearman }\left(r_{s}\right) \text { correlation coefficients. }\end{array}$} \\
\hline \multicolumn{5}{|c|}{$\begin{array}{l}\text { UE = uncontrolled eating; } \mathrm{EE}=\text { emotional eating; } \mathrm{CR}=\text { cognitive restraint; } \mathrm{TC}=\text { total cholesterol; } \mathrm{TG}= \\
\text { triglycerides; } \mathrm{LDL}-\mathrm{C}=\text { low density lipoprotein cholesterol; } \mathrm{HDL}-\mathrm{C}=\text { high density lipoprotein cholesterol; } \\
\text { FM\% = fat mass percentage; } \mathrm{WC}=\text { waist circumference. }\end{array}$} \\
\hline
\end{tabular}

The teenager with greatest CR score at the end of the study also had the greatest reduction in BMI zscore, although this result was not statistically significant.

Comparing the levels of PYY hormone with the other variables in study, we observed a significantly negative correlation between PYY and BMI $(r=-0.421 ; p=0.002)$, and PYY and BMI $z$-score $(r=-0.340 ; p=$ 0.001). However, there was no significant correlation between the hormone and any EB type (Table 3 ).

Table 3

Association between PYY hormone levels with other variables by Pearson ( $r)$ or Spearman $\left(r_{s}\right)$ correlation coefficients.

\begin{tabular}{|c|c|c|c|c|}
\hline PYY vs variables & $\begin{array}{l}\text { TO } \\
\text { C coef }\end{array}$ & $\begin{array}{l}\text { T1 } \\
\text { C coef }\end{array}$ & $\begin{array}{l}\text { T2 } \\
\text { C coef }\end{array}$ & $\begin{array}{l}\text { Time variation 1-3 }(\Delta) \\
\text { C coefi }\end{array}$ \\
\hline BMI & 0.041 & 0.129 & 0.018 & $-0.421 * \star$ \\
\hline BMI z-score & 0.105 & 0.144 & -0.037 & $-0.340 *$ \\
\hline UE & -0.067 & -0.054 & -0.170 & -0.053 \\
\hline CR & 0.120 & 0.138 & -0.220 & -0.039 \\
\hline $\mathrm{EE}$ & 0.185 & -0.178 & 0.215 & 0.131 \\
\hline \multicolumn{5}{|c|}{ ** $p<0.01-$ Significant Inverse association, the lower the BMI, the higher the PYY hormone levels } \\
\hline \multicolumn{5}{|c|}{${ }^{*} \mathrm{p}<0.05$-Significant inverse association, the lower the BMI z-score, the higher the PYY hormone levels } \\
\hline \multicolumn{5}{|c|}{$\mathrm{UE}=$ uncontrolled eating; $\mathrm{EE}=$ emotional eating; $\mathrm{CR}=$ cognitive restraint; $\mathrm{BMI}=$ body mass index } \\
\hline
\end{tabular}

For the associations between PYY hormone levels and other study variables, there was only a significant association between changes in BMI and PYY from T0 to T2 ( $r=-0.421 ; p=0.002)$, and for BMI z-score ( $r$ $=-0.340 ; p=0.001)($ Table 3$)$.

\section{Discussion}


Our study showed that weight loss recommendations based on quantitative and qualitative food targets and a reduction in sedentary behavior yielded weight loss over 12 months of follow-up. Lifestyle interventions are the predominant recommendation for childhood and adolescent weight loss, and those who prioritize nutritional counseling and increases in physical activity - with favorable changes in EB have been successful with weight loss and maintenance as adults (11).

Lang and Froelicher (2006) suggest that promoting healthier eating habits and reducing sedentary behavior yield favorable change in EB (12). The consumption of foods rich in simple carbohydrates and saturated and trans fats cause changes in steady- state in fasting. Therefore, we can infer that our cohort may have reduced their intake of foods that increase blood glucose levels, insulin and TG. The EB of an obese individual may be connected to easy access to energy-dense and highly palatable foods. During follow-up with patients, reductions intake in sugar, fat and ultra processed foods were emphasized. Studies have shown that reducing intake of sweetened beverages is associated with a decrease in child and adolescent overweight, and that reducing consumption of fast foods is promising for weight loss $(13,14)$.

Our results may suggest that the stage of lipolysis may be related to a favorable change in EB because participants display more self-control (CR) and less EE when serum levels of insulin, glucose and TG are reduced. According to Maclean et al. (2011), a decline in fasted insulin is evidence of weight loss and calorie restriction. Schwartz et al. (1997) indicate that the weight loss is characterized by low levels of insulin $(15,16)$. Brogan et al. $(2012)$ argue that CR can be a successful strategy for weight loss $(17)$.

The amount of weight loss needed to improve the quality of life of obese adolescents has not been established. However, studies show that a reduction in BMI z-score score $>0.5$ reduces cardiovascular risk factors and insulin resistance (18). Even with modest weight loss, the intervention had a significant impact on the overall health of these adolescents.

For Keränen et al. (2009), changes in EB do not depend on whether the recommendations are intensive or short-term (19). Our findings showed significant results after 12 months of follow-up, corroborating findings from other interventions with 6-12 months of follow-up (20).

Reduced BMIs were associated with changes in circulating levels of PYY. The present study shows that PYY reduces in concomitance with weight loss or with food restriction.These; findings corroborate other studies claiming that PYY helps reduce appetite, body weight and adiposity $(21,22)$. Although we have no significant association between WC, TC and TG with PYY, the lowering of these parameters over time suggests a reduction in cardiometabolic risk for adolescents (23).

TFEQ-21 is more often used in adults than in obese adolescents. Studies in adults have shown that the high susceptibility to overeat (uncontrolled eating) combined with low CR is associated with increased body weight (24). Our research found no significant association between BMI and UE and CR behaviors, but we did find that those with lower UE and higher CR had better glucose results over the 12 month follow-up. 
Contrary to studies by Hainer et al. (2006) and Bryant et al., (2008) we found no significant association between UE, CC, EE, BMI and other anthropometric parameters $(25,26)$. We assume that the absence of a correlation is due to the sample size, because UE and EE scores decreased among male and female participants while CR scores increased. Higher CR scores increases the likelihood of weight loss maintenance in individuals with excess weight (19).

Our study found no associations between the biological responses of the PYY hormone and the behavioral phenotypes UE, CR and EE, but we did see a favorable change in ED over 12 months. The adolescents in our study acquired a more controlled EB over 1 year. According to some studies, individuals with UE and EE are unsuccessful in weight loss and maintenance $(19,27)$.

Food intake is regulated by glucose, amino acids, hormones and neuropeptides. Insulin signals adiposity within the brain and in central nervous system influencing energy homeostasis through food intake and body weight (28). Adipose tissue increases the demand for insulin and, in obese patients, can cause heightened levels of blood glucose, favoring lipogenesis. Patients who decreased their UE and EE scores the most were, respectively, those who lowered their serum levels of insulin and glucose the most. The patients who lowered their insulin and TG the most were those who increased their CR score the most.

An increase in CR score can denote self-control over food intake. However, we should emphasize that our results on EB do not imply causality; although it is possible to infer that CR, UE and EE behaviors have an impact on food intake and weight loss.

\section{Conclusions}

The effect of gender on emotional eating is controversial in the existing research. In our cohort, there was no significant difference between the emotional eating scores of male and female participants. The lack of significant changes in glucose and dyslipidemic profiles is perhaps due to the short length of the weight loss program. We assume that the lack of significant changes in lipid profile was also due to short-duration of the weight loss program. After weight loss, there was a significant correlation between UE and lipid profile, which is in line with the conclusions of Hainer et al. (2006), and Hays et al. (2002) $(25,29)$.

In conclusion, our results showed that after monitoring weight loss for a year, adolescents had more control over their eating, increased levels of PYY anorectic hormone and reduced weight.

\section{List Of Abbreviations}

BMI - body mass index

CR - Cognitive Restraint

EB - Eating behavior 
EE-Emotional Eating

HDL-C - high density lipoprotein cholesterol

HOMA-IR - homeostasis model assessment

LDL-C- low density lipoprotein cholesterol

PYY - peptide tyrosine tyrosineTC - total cholesterol

TFEQ-21 - Three-Factor Eating Questionnaire

TG - triglycerides

UE - Uncontrolled Eating

WC - waist circumference

\section{Declarations}

\section{ETHICS APPROVAL AND CONSENT TO PARTICIPATE}

All study procedures were reviewed and approved by the ethics committee of the Hospital de Clínicas de Porto Alegre, under protocol 130190. All participants signed the Free, Prior and Informed Consent Form agreeing to participate in the proposed study.

CONSENT FOR PUBLICATION

Not applicable

\section{AVAILABILITY OF DATA AND MATERIALS}

The data that support the findings of this study are available on request from the corresponding author. COMPETING INTERESTS

No conflicts of interest were declared. The authors have no financial or non-financial relationships to disclose.

\section{FUNDING}

S.P.F received funding from the Universidade Federal do Rio Grande do Sul (UFRGS) and Hospital de Clínicas de Porto Alegre (HCPA) to conduct the study.

\section{AUTHORS CONTRIBUTIONS}


S.P.F. was a major contributor collecting data, interpreting results and writing the manuscript. J.A. assisted in interpreting results and writing the manuscript and Z.E.A.S. and E.D.D.M. assisted in supervision. All authors interpreted results, critically revised the manuscript, and approved the final version.

\section{ACKNOWLEDGEMENTS}

The authors thank the Child obesity clinic (AMO) of the Hospital de Clínicas de Porto Alegre (HCPA) and the study investigators, participants, and personnel.

\section{References}

1. Ng M, Fleming T, Robinson M, et al. Global, regional, and national prevalence of overweight and obesity in children and adults during 1980-2013: a systematic analysis for the Global Burden of Disease Study 2013. 2014;384(9945):766-781.

2. Aiello AM, Marques de Mello L, Souza Nunes M, Soares da Silva A, Nunes A. Prevalence of Obesity in Children and Adolescents in Brazil: A Meta-analysis of Cross-sectional Studies. Curr Pediatr Rev. 2015;11(1):36-42.

3. Stunkard AJ, Messick S. The three-factor eating questionnaire to measure dietary restraint, disinhibition and hunger. J Psychosom Res. 1985;29(1):71-83.

4. Tanofsky-Kraff M, Shomaker LB, Olsen C, et al. A prospective study of pediatric loss of control eating and psychological outcomes. J Abnorm Psychol. 2011;120(1):108-118.

5. Dietrich A, Hollmann M, Mathar D, Villringer A, Horstmann A. Brain regulation of food craving: relationships with weight status and eating behavior. Int J Obes (Lond). 2016;40(6):982-989.

6. Marshall WA, Tanner JM. Growth and physiological development during adolescence. Annu Rev Med. 1968;19:283-300.

7. de Onis M, Onyango AW, Borghi E, Siyam A, Nishida C, Siekmann J. Development of a WHO growth reference for school-aged children and adolescents. Bull World Health Organ. 2007;85(9):660-667.

8. de Mello ED, Luft VC, Meyer F. [Individual outpatient care versus group education programs. Which leads to greater change in dietary and physical activity habits for obese children?]. J Pediatr (Rio J). 2004;80(6):468-474.

9. Barlow SE, Dietz WH. Management of child and adolescent obesity: summary and recommendations based on reports from pediatricians, pediatric nurse practitioners, and registered dietitians. 2002;110(1 Pt 2):236-238.

10. Fernandes SP, Conterato EV, de Mello ED. Manejo do paciente obeso pediátrico na atenção primária: proposta de uma abordagem terapêutica prática. Rev HCPA; 2012:32(4)461472. 
11. Dombrowski SU, Knittle K, Avenell A, Araújo-Soares V, Sniehotta FF. Long term maintenance of weight loss with non-surgical interventions in obese adults: systematic review and meta-analyses of randomised controlled trials. 2014;348:g2646.

12. Lang A, Froelicher ES. Management of overweight and obesity in adults: behavioral intervention for long-term weight loss and maintenance. Eur J Cardiovasc Nurs. 2006;5(2):102-114.

13. James J, Thomas P, Kerr D. Preventing childhood obesity: two year follow-up results from the Christchurch obesity prevention programme in schools (CHOPPS). 2007;335(7623):762.

14. Muckelbauer R, Libuda L, Clausen K, Reinehr T, Kersting M. A simple dietary intervention in the school setting decreased incidence of overweight in children. Obes Facts. 2009;2(5):282-285.

15. Maclean PS, Bergouignan A, Cornier MA, Jackman MR. Biology's response to dieting: the impetus for weight regain. Am J Physiol Regul Integr Comp Physiol. 2011;301(3):R581-600.

16. Schwartz MW, Seeley RJ. Seminars in medicine of the Beth Israel Deaconess Medical Center. Neuroendocrine responses to starvation and weight loss. N Engl J Med. 1997;336(25):1802-1811.

17. Brogan A, Hevey D. Eating styles in the morbidly obese: restraint eating, but not emotional and external eating, predicts dietary behaviour. Psychol Health. 2013;28(6):714-725.

18. Reinehr T, Kleber M, Toschke AM. Lifestyle intervention in obese children is associated with a decrease of the metabolic syndrome prevalence. 2009;207(1):174-180.

19. Keränen AM, Savolainen MJ, Reponen $A H$, et al. The effect of eating behavior on weight loss and maintenance during a lifestyle intervention. Prev Med. 2009;49(1):32-38.

20. Monasta L, Batty GD, Macaluso A, et al. Interventions for the prevention of overweight and obesity in preschool children: a systematic review of randomized controlled trials. Obes Rev. 2011;12(5):e107118.

21. Viskaal-van Dongen M, Kok FJ, de Graaf C. Eating rate of commonly consumed foods promotes food and energy intake. 2011;56(1):25-31.

22. Batterham RL, Heffron $H$, Kapoor $S$, et al. Critical role for peptide $Y Y$ in protein-mediated satiation and body-weight regulation. Cell Metab. 2006;4(3):223-233.

23. Reaven GM. The metabolic syndrome: time to get off the merry-go-round? J Intern Med. 2011;269(2):127-136.

24. Dykes J, Brunner EJ, Martikainen PT, Wardle J. Socioeconomic gradient in body size and obesity among women: the role of dietary restraint, disinhibition and hunger in the Whitehall II study. Int $J$ Obes Relat Metab Disord. 2004;28(2):262-268.

25. Hainer V, Kunesova M, Bellisle F, et al. The Eating Inventory, body adiposity and prevalence of diseases in a quota sample of Czech adults. Int J Obes (Lond). 2006;30(5):830-836.

26. Bryant EJ, King NA, Blundell JE. Disinhibition: its effects on appetite and weight regulation. Obes Rev. 2008;9(5):409-419.

27. Pacanowski CR, Senso MM, Oriogun K, Crain AL, Sherwood NE. Binge eating behavior and weight loss maintenance over a 2-year period. J Obes. 2014;2014:249315. 
28. Takei N, Furukawa K, Hanyu O, Sone H, Nawa H. A possible link between BDNF and mTOR in control of food intake. Front Psychol. 2014;5:1093.

29. Hays NP, Bathalon GP, Roubenoff R, Lipman R, Roberts SB. The association of eating behavior with risk for morbidity in older women. J Gerontol A Biol Sci Med Sci. 2002;57(2):M128-133. 